\title{
Qualitative Investigating on the Effect of Fine Water Spray and Its Orientation in Mitigating Vapour Cloud Explosions
}

\author{
Stephen. A. Johnson", Ghasem G Nasr², Amir Nourian², Muhammad K Abba² \\ ${ }^{1}$ Department of Gas Engineering, Specialist Gas Assessment Services (SGAS), Blackpool, \\ UK \\ ${ }^{2}$ Spray Research Group (SRG), School of Science, Engineering and Environment (SEE), \\ University of Salford, Manchester, UK \\ ${ }^{*}$ Corresponding author email: $\underline{\text { a.nourian@salford.ac.uk }}$
}

\begin{abstract}
For past decades, various industries, such as oil and gas, have come to realisation that using water-based spraying systems, with fine very fine droplets should be the preferred option in mitigating and suppressing the thermal explosions. Previous studies have mainly focused on improving efficiency of flame mitigation by utilising existing water spray systems with droplets greater than 200 microns. This paper provide analysis in the utilisation of fine water sprays (between 10 to 30 microns) using Spill Return Atomisers (SRAs) to mitigate slow moving flames within the range of 5 to $30 \mathrm{~m} / \mathrm{s}$. The flame produced by a mixture of methane and air at four different Equivalent Ratios (ER, $\varphi$ ) between 0.61 to 1.06. Three different SRAs (Type $A, B$, and C) with variation in exit orifice are, also, placed in two different orientations (counter and parallel flow). The results indicate that by using single spray in both directions, atomisers Type-A and $B$ are not capable of mitigating the flame at different equivalent ratio of methane/air mixture. However, atomiser Type-C shows full mitigation $\varphi 0.61$. Therefore, to mitigate the flame with $\varphi>0.61$, the density and volume flux of the water droplets within the flame path should be increased.
\end{abstract}

\section{Keywords}

flame mitigation, fire suppression, fine water spray, spill return atomiser (SRA), methane-air mixture.

\section{Introduction}

Gas and vapour cloud explosions are undoubtedly unavoidable. This can be attributed to the reactivity and flammability of the reacting species. Human error and engineering defects are factors that can increase the chances of the occurrence of these destructive phenomena. Explosions are defined by the rate of simultaneous expansion as the participating species react to form products. This thermal expansion can produce massive expansions and produce a wide range of field overpressures that can reach up to 50 atmospheres [1]. For several highrisk sites such as oil and gas platforms, the potential for an explosion can significantly be reduced by simply following safety precautions and adopting the appropriate design criteria. This aversion to explosions can be exerted by providing a safety risk management process, such as Control of Major Accident Hazards Regulations (COMAH) [2], which is legal and enforceable in the UK. Usually, there will be a prospect to retrofit existing sites by altering site design and layout to improve the accessibility or installing mitigating systems in places like water deluge or explosion venting procedures. Many research studies in utilising water sprays, with particle sizes of D32 $\geq 100$ microns, for suppressing and mitigating the explosion have been extensively carried out in the past as [3-10]. Additionally, they reported a sufficient amount of liquid volume flux to mitigate a high-speed propagating flame (100 to $2000 \mathrm{~m} / \mathrm{s}$ ), representing the flame produced in high loss incidents caused by flame acceleration and significant-high overpressures. The blast shockwave upstream of the combustion trail can 
provide the dynamic forces required to further break up the water droplets smaller particles due to the accelerated flame speeds. Also, these works reported that suppression of combustion or mitigation of the flame activity only occurred as a result of the dynamic forces created by the blast wave overcoming the surface tension forces in the water droplets. Fine mists formed by the larger droplets' hydrodynamic breakup, owing to the upstream blast, could then progress through the flame. A high degree of successful mitigation can thus be achieved, provided that, within the reaction zone of the flame, there were droplets with enough liquid volume flux $\left(\mathrm{Q}_{\mathrm{f}}\right)$ and sufficient residence time $(\mathrm{t})$. This will facilitate flame suppression or mitigation of combustion. The previous studies reached a collective conclusion that water spray is a very effective method to suppress and/or mitigate the gas and vapour cloud explosions, even at very high flame speeds.

It has become clear that previous research studies specifically relied on the subsequent break up of water droplets into fine mist as a result of the upstream blast. To this effect, the forces holding water droplets together should be smaller than those generated by the blast shockwave. In many cases and more specifically within an unconfined area, overpressures are extremely low, and the water droplets cannot break up further, which keep them within their original size. In another study, Lane [11] presented a relationship between droplet diameter and the critical velocity needed to overcome the intrinsic forces i.e. surface tension, which hold droplets together (see Equation 1).

In many cases, in explosions that occur in open environments, the pressures generated are so low that they have little effect on the further decomposition of water droplets and thus retain their original geometry.

$$
\left(V_{c}\right)^{2} d=0.612 \times 10^{6} \frac{\mathrm{m}^{3}}{\mathrm{~s}^{2}}
$$

$V c$ is the critical relative gas stream velocity for droplet break up $(\mathrm{m} / \mathrm{s})$, and $d$ is the droplet diameter $(\mu \mathrm{m})$. Furthermore, the water surface tension is considered to $73.10 \mathrm{mN} / \mathrm{m}$ and the density of the gas mixture is taken to be $1.2 \mathrm{~kg} / \mathrm{m}^{3}$. Lane's formula is consistent with a critical Weber number stated by many authors of required for droplet break up [ $\underline{4}, \underline{11}, \underline{12}$. Although other studies have all identified the conditions required to mitigate high-speed explosions, the objective of the current study is mitigation of slow flame speeds ( $\leq 30 \mathrm{~m} / \mathrm{s}$ ) utilising small enough $(\leq 30 \mu \mathrm{m})$ water droplets to directly remove the heat from the flame thus to supress or mitigate the flame. A purpose-built 'Flame Propagation and Mitigation Rig (FPMR)' was used in this study to produce flame from a mixture of methane and air at an equivalent ratio $(E R, \varphi)$ of 0.61 to represent lean combustion. Different Spill Return Atomisers (SRA), with variation in exit orifice, were positioned in two different directions than the flame direction (parallel or counter) and placed as either single or two overlapping sprays.

\section{Experimental Setup}

Figure 1 shows the Spill Return Atomiser (SRA) which was used in this study and it was developed by Nasr et al. [13] with capability of producing fine mist water droplets [13-16]. in order to investigate the effect of spray performance (i.e., flow rate, liquid volume flux, spray cone angle, and droplet size) on mitigating the flame, the original nozzle (Type-A with $0.3 \mathrm{~mm}$ exit orifice) was additionally modified to Type-B (with $0.5 \mathrm{~mm}$ exit orifice) and Type- $\mathrm{C}$ (with 0.8 $\mathrm{mm}$ exit orifice). 


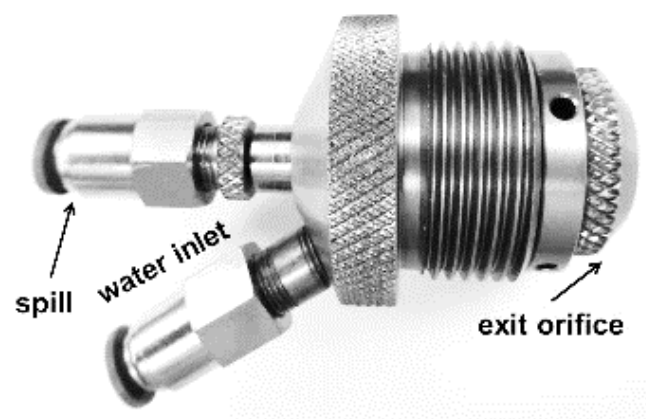

Figure 1. Assembled and components of the SRA [15]

The spray characterisation of these nozzles' configuration carried out at different water supply pressure (from 5 to $14 \mathrm{MPa}$ ) and the spray cone angle, droplet size, and the liquid volume flux were measured and analysed by using Phase Doppler Anemometry (PDA) and image processing.

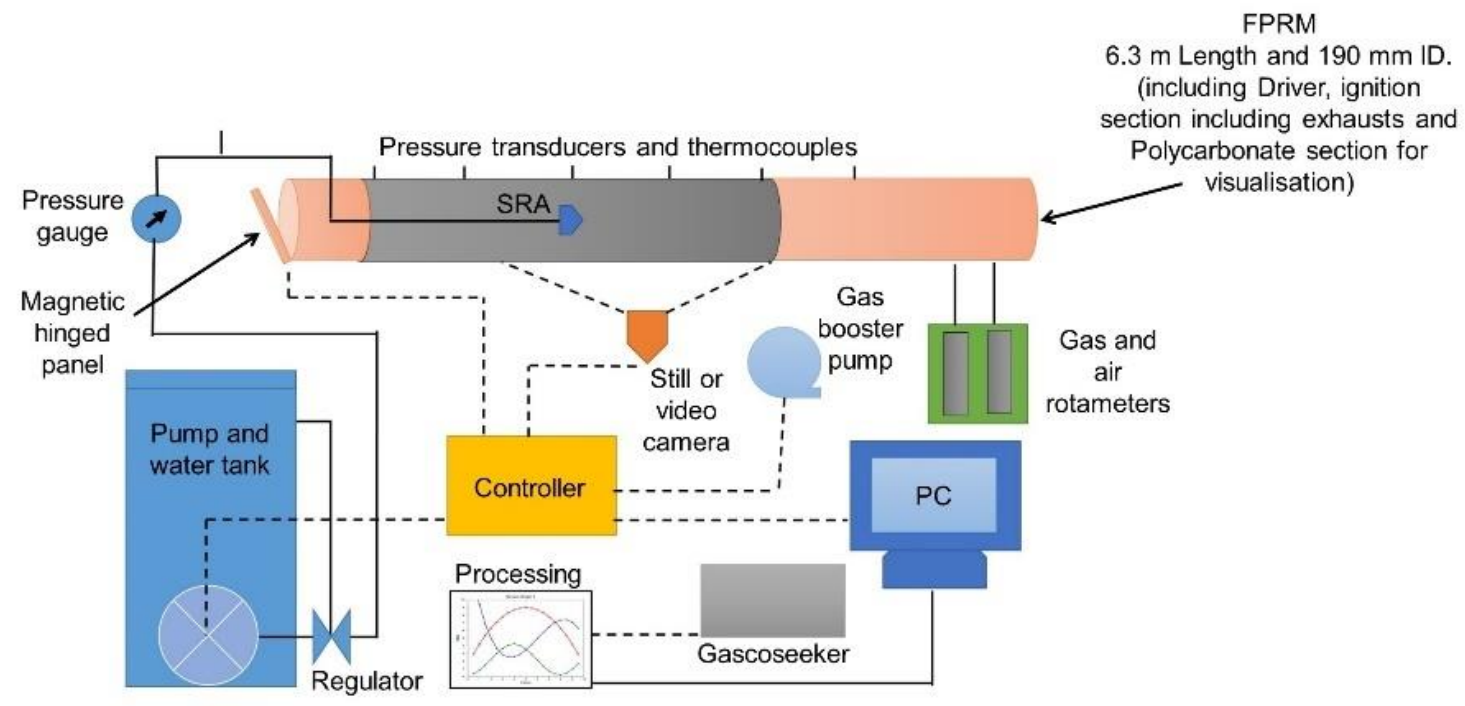

Figure 2. Schematic diagram of the experimental setup

All these tests conducted in atmospheric condition as well as within the FPMR in the absence of the flame or the mixture of methane/air and the full details have been reported in previous studies $[13,15]$. Figure 2 shows the schematic diagram of the experimental setup.

\section{Results and Discussion}

Table 1 shows the summary of the spray characterisation of SRA Type-A, B, and C with water supply pressure of $13 \mathrm{MPa}$.

Table 1 - Summary of dynamic spray and droplet measurements using a water pressure of $13 \mathrm{MPa}[\underline{15}, \underline{16}]$

\begin{tabular}{c|ccc}
\hline & Type-A & Type-B & Type-C \\
\hline Cone angle, $\theta$ (degree) & 34.7 & 42.7 & 49.2 \\
Average droplet size, D32 (microns) & 17 & $26-34$ & $29-39$ \\
Average liquid volume flux, Qf (cm3/s/cm2) & 0.011 & $0.024-0.044$ & $0.039-0.047$
\end{tabular}


As described before, the present work's objective is to investigate the effect of spray orientation in relation to the flame direction at the different mixture of methane/air combustion. Therefore, each SRA nozzles (Type-A, B and C) were placed in two different orientation relative to the propagating flame direction (counter or parallel flow), as shown in Figure 3.

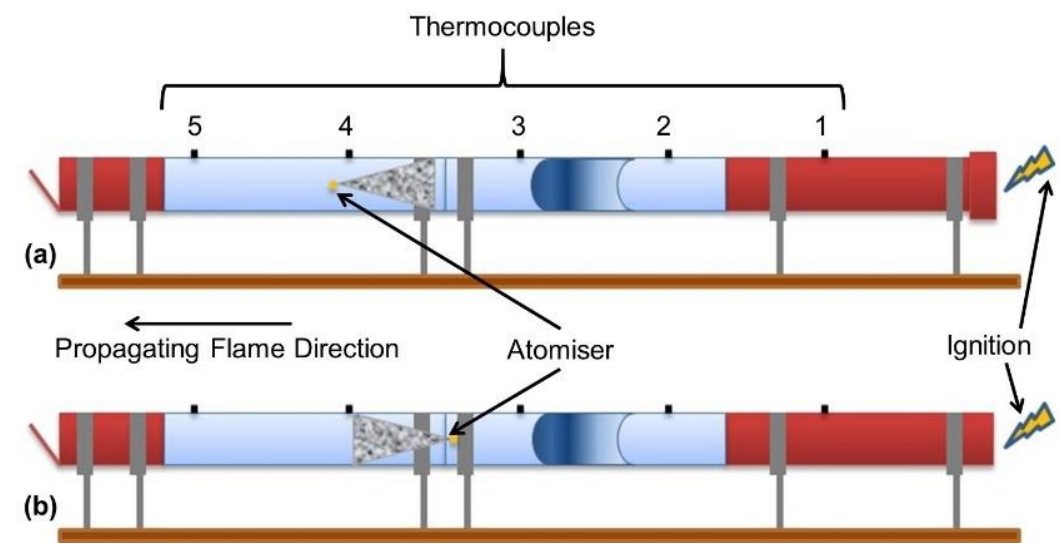

Figure 3. Demonstration of spray orientation compared to the flame direction: (a) counter, and (b) parallel flow.

Figure 4 shows the flame generates at different methane/air mixture in which the SRA nozzle is located, but there is no water spray (called 'dry' condition).

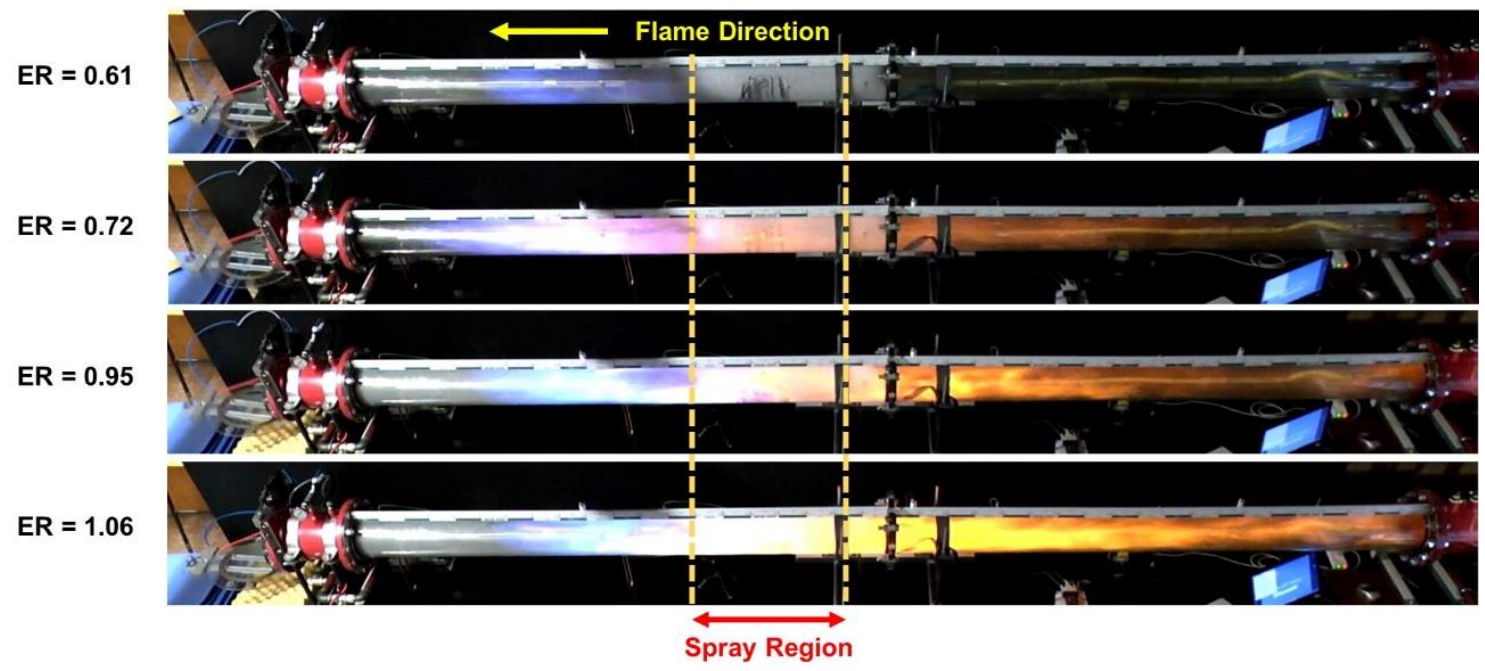

Figure 4: Flame propagating with SRA nozzles in placed but without spraying (dry condition).

This is to benchmark the experiments to identify the effect of water spray on flame mitigation. As can be seen, in all different ERs, flame travels from the ignition region, and it approaches the spray region where a single SRA nozzle (Type-A, B, or C) attached, and water droplets (from supply pressure of $13 \mathrm{MPa}$ ) engages with it. Subsequently, the flame is moving further and exit the pipe through an opened-hinged located at the end of the tube.

\section{Single SRA Type-A}

Figure 5 illustrates the qualitative analysis of the flame propagating by using SRA Type-A located in two different orientations compared to the flame direction. It can be seen that, in counterflow spray and for all ERs, there is no evidence of visual break, dark region or colour changes in the flame and the flame can be observed downstream of the spraying region which 
means this particular configuration was unsuccessful to mitigate the flame. This is due to the fact that SRA Type-A provides a very small droplet size and liquid volume flux that the water droplets could not interact with the flame heat and reduce the temperature.
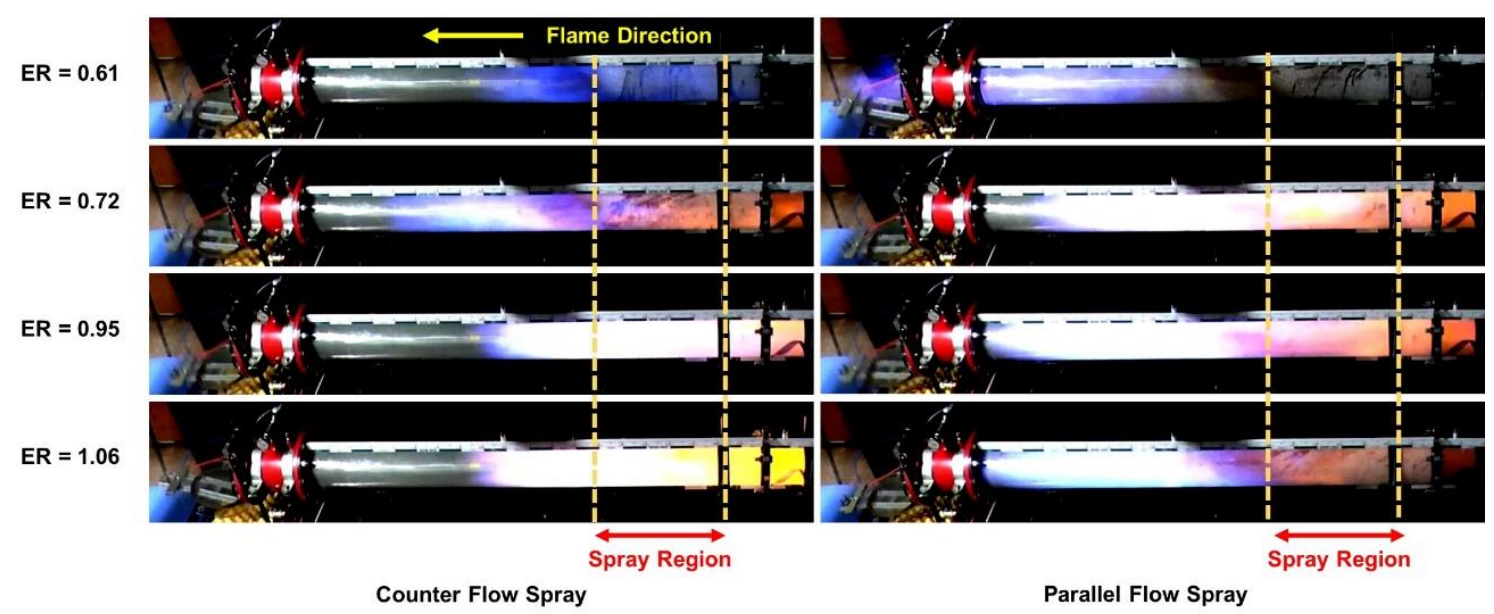

Figure 5: Flame propagating using SRA Type-A with counter and parallel flow spray compared to the flame direction.

The images of parallel flow spray are indicating almost the same argument as the counter flow. However, it should be noted that, there is a visual break (or dark region) within the spraying region of ER 0.61 which can show a very small effect of the spray in mitigating and disturbing the flame.

\section{Single SRA Type-B}

Figure 6 reveals the flame's images for a different mixture of the methane-air mixture and using SRA Type-B. In all cases, the flame can be seen after passing the spraying region, which means that this nozzle configuration was also enabled to mitigate the flame. However, analysing the images of counter flow spray, a break up region (dark area) within the combustion zone can be observed. This is indicating a level of suspension in the combustion activity. This can be related to the spray performance of SRA Type-B which has larger water droplets and higher liquid volume flux compared to SRA Type-A.
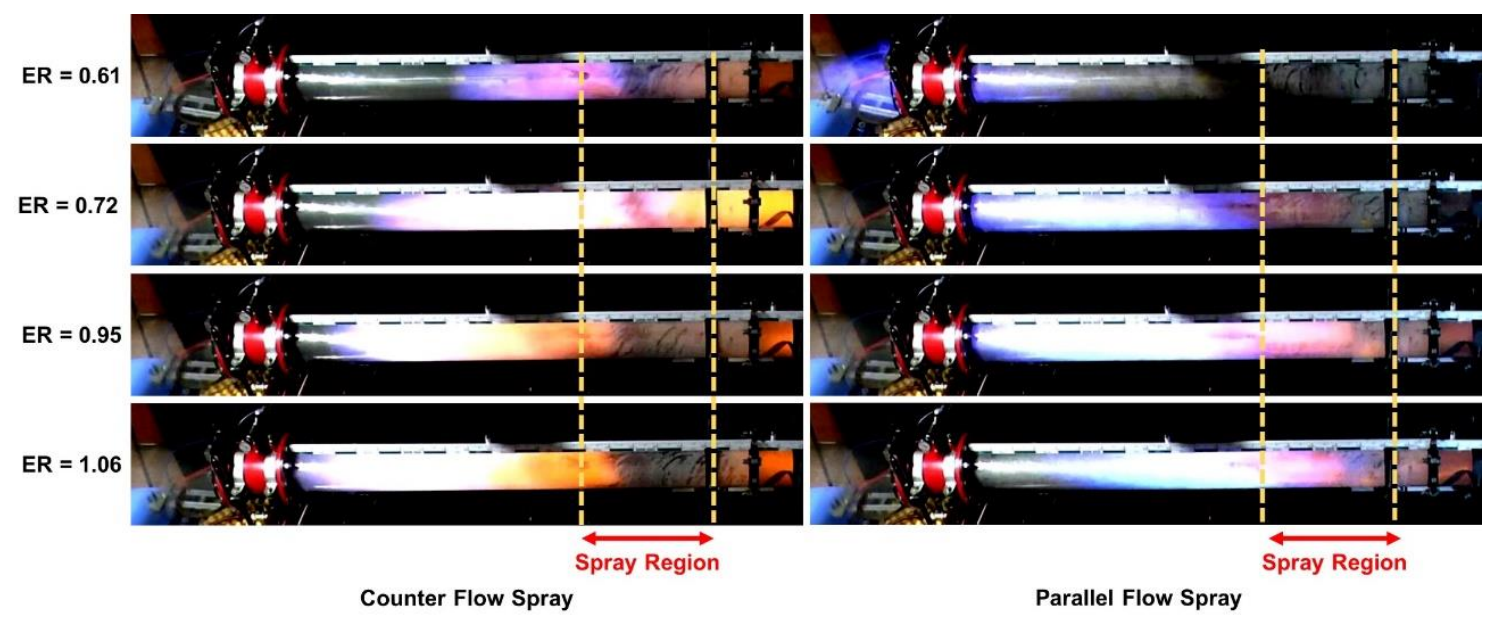

Figure 6: Flame propagating using SRA Type-B with counter and parallel flow spray compared to the flame direction. 
As shown in images for parallel flow spray, the flame within the spraying region for ER 0.61 and 0.72 has been disturbed and a dark region can be seen, but the flame can still be observed in ER 0.95 and 1.06. However, it is clear that the flame colour has changed which shows that the water droplets are trying to interact with the flame but there is insufficient energy to mitigate it.

\section{Single SRA Type-C}

Figure 7 shows a qualitative image taken from flame propagating the different methane/air mixtures with using single SRA Type-C. As it shows, for ER 0.61 and both spray direction (counter and parallel flow), the flame did not pass the wet region where the nozzle is located. Subsequent ignition of the downstream flammable mixture did not occur and the flame was mitigated. However, analysing the flame images for other ERs, indicate the combustion flame passed though the spraying region with a dark breaking up region in the flame which shows some areas of a suspension of combustion. Moreover, for the mixture of methane/air with ER 1.06 in counter flow spray, the flame passed through the wet region, all the combustion process was immediately supressed by the water spray.

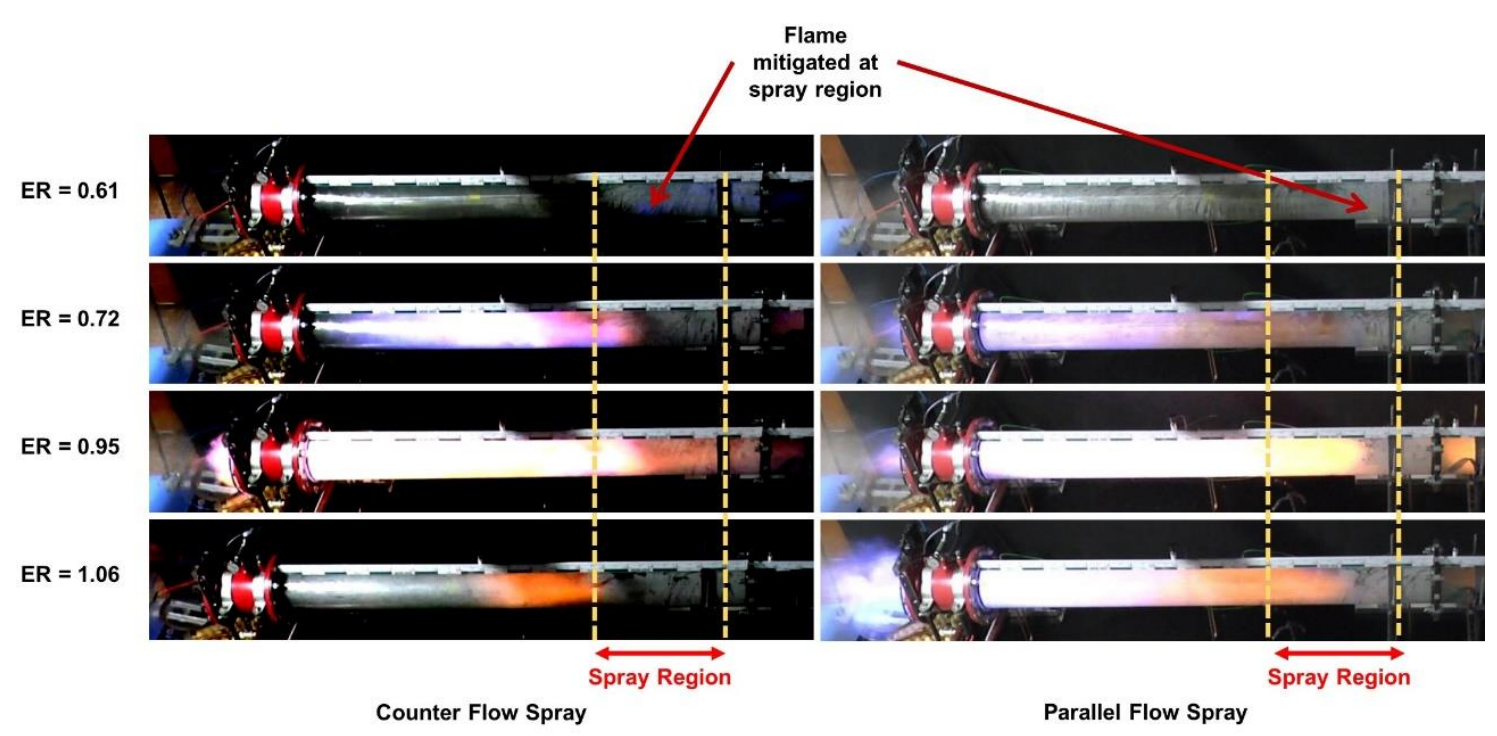

Figure 7: Flame propagating using SRA Type-C with counter and parallel flow spray compared to the flame direction.

Therefore, it can be argued that SRA Type-C shows promising results since it provides better spray droplet size, liquid volume flux, and spray cone angle compared to other two nozzles.

\section{Conclusions}

The combustion mitigation potential of three different SRA in two different orientations (counter and parallel flow) compared to the flame direction at four mixtures of methane/air (ranging from lean to rich combustion) has been clearly demonstrated discussed in this study. The results indicate that:

- The droplet size and liquid volume flux from SRA Type-A are insufficient to mitigate the flame.

- Although the spray from SRA Type-B could disturb the flame when it passes through the spraying region, it could not fully mitigate the combustion at all different ERs.

- Spray from SRA Type-C could successfully stop the flame passing through the pipe at ER 0.61. 


\section{Future Work}

The present work results demonstrate that to mitigate a combustion flame for the mixture of methane/air at ER greater than 0.61 , the water droplet density and liquid volume within the flame passage should increase. Therefore, future work will include the experimental and numerical analysis of the correct combination of more than one nozzle, leading to the flame's full mitigation.

\section{References}

[1] G. Nasr and N. Connor, "Natural Gas Engineering and Safety Challenges," Springer International Publishing, p. 402, 2014, doi: https://doi.org/10.1007/978-3-319-08948-5.

[2] Health and Safety Executive (HSE), "The Control of Major Accident Hazards Regulations 2015 (3rd edition)," 2015. [Online]. Available: https://www.hse.gov.uk/pubns/priced/l111.pdf.

[3] M. J. Sapko, A. L. Furno, and J. Kuchta, Quenching methane-air ignitions with water sprays. Dept. of the Interior, Bureau of Mines, 1977.

[4] R. Zalosh and S. Bajpai, "Water fog inerting of hydrogen-air mixtures," International Nuclear Information System (INIS), vol. 16, no. 23, 1982.

[5] P. Cornin and M. J. Wickens, "A large scale experimental study of the conditions required to sustain high speed flame propagation in a flammable vapour cloud," 1986.

[6] C. Catlin, "Scale effects on the external combustion caused by venting of a confined explosion," Combustion and Flame, vol. 83, no. 3, pp. 399-411, 1991, doi: https://doi.org/10.1016/0010-2180(91)90086-Q.

[7] D. Johnson, P. Sutton, and M. Wickens, "Scaled experiments to study vapour cloud explosions," Process Safety and Environmental Protection, vol. 69, pp. 76-84, 1991.

[8] K. Van Wingerden and B. Wilkins, "The influence of water sprays on gas explosions. Part 1: water-spray-generated turbulence," Journal of loss prevention in the process industries, vol. 8, no. 2, pp. 53-59, 1995, doi: https://doi.org/10.1016/09504230(95)00002-I.

[9] K. Van Wingerden, B. Wilkins, J. Bakken, and G. Pedersen, "The influence of water sprays on gas explosions. Part 2: mitigation," Journal of Loss Prevention in the Process Industries, vol. 8, no. 2, pp. 61-70, 1995, doi: https://doi.org/10.1016/09504230(95)00007-N.

[10] J. W. Fleming and R. S. Sheinson, "Chapter 4: Aerosol Properties," U.S. Naval Research Laboratory, $2007 . \quad$ [Online]. Available: https://www.nist.gov/system/files/documents/el/fire research/chapter-4.pdf

[11] W. Lane, "Shatter of drops in streams of air," Industrial \& Engineering Chemistry, vol. 43, no. 6, pp. 1312-1317, 1951, doi: https://doi.org/10.1021/ie50498a022.

[12] R. Harris and M. Wickens, "Understanding vapour cloud explosions-an experimental study," Publ./Institution of gas eng., 1989.

[13] G. Nasr, A. Yule, J. Stewart, A. Whitehead, and T. Hughes, "A new fine spray, low flowrate, spill-return swirl atomiser," Proceedings of the Institution of Mechanical Engineers, Part C: Journal of Mechanical Engineering Science, vol. 225, no. 4, pp. 897-908, 2011, doi: https://doi.org/10.1243\%2F09544062JMES2675.

[14] J. Stewart, "Design and development of a novel spill return atomiser (SRA) disinfection system for healthcare environments," PhD, School of Computing, Science and Engineering (CSE), University of Salford, 2011.

[15] S. A. Johnston, G. G. Nasr, and A. Nourian, "Utilisation of fine water sprays in explosion mitigation: cold trial," vol. 30, no. 6, pp. 431-450, 2020-10-09 2020, doi: https://doi.org/10.1615/AtomizSpr.2020034356.

[16] S. A. Johnston, "Mitigation of gas and vapour cloud explosions using fine water sprays," PhD, School of Computing, Science and Engineering (CSE), University of Salford, 2015. 